\title{
Arbitrary discontinuities in finite elements
}

\author{
T. Belytschko*,t, , N. Moës ${ }^{\S}$, S. Usui ${ }^{\Uparrow}$ and C. Parimi ${ }^{\|}$ \\ Department of Mechanical Engineering, Northwestern University, 2145 Sheridan Road, \\ Evanston, IL 60208, U.S.A.
}

\begin{abstract}
SUMMARY
A technique for modelling arbitrary discontinuities in finite elements is presented. Both discontinuities in the function and its derivatives are considered. Methods for intersecting and branching discontinuities are given. In all cases, the discontinuous approximation is constructed in terms of a signed distance functions, so level sets can be used to update the position of the discontinuities. A standard displacement Galerkin method is used for developing the discrete equations. Examples of the following applications are given: crack growth, a journal bearing, a non-bonded circular inclusion and a jointed rock mass.
\end{abstract}

KEY WORDS: finite elements; fracture; fasteners; jointed rock

\section{INTRODUCTION}

This paper unifies and extends the modelling of functions with arbitrary discontinuities and discontinuous derivatives in finite elements first proposed in References [1-4]. The discontinuities are completely independent of the finite element mesh: they can cross elements in any manner. This is particularly useful for evolution problems with moving discontinuities, such as solidification, other phase changes, cracks, shear bands and joints in rock. In problems involving the evolution and motion of discontinuities, it avoids the need for remeshing. It also provides a powerful tool for modelling unusual problems in engineering, such as bolts, joints, etc. In these problems, it avoids the need for sliding interfaces that conform to the mating meshes.

The techniques for approximating discontinuities are based on the concepts described in References $[1,2]$. We emphasize modelling in finite element methods, but these methods also apply to meshfree approximations such as the element-free Galerkin method, EFG [5].

\footnotetext{
* Correspondence to: Ted Belytschko. Mechanical Engineering Department, Northwestern University, 2145 N Sheridan, Rm 224, Evanston, IL 60208-3111, U.S.A.

$\dagger$ Walter P. Murphy, Professor

‡E-mail: t-belytschko@northwestern.edu

$\S$ Research Assistant Professor

ब Graduate Student

| Graduate Student
} 


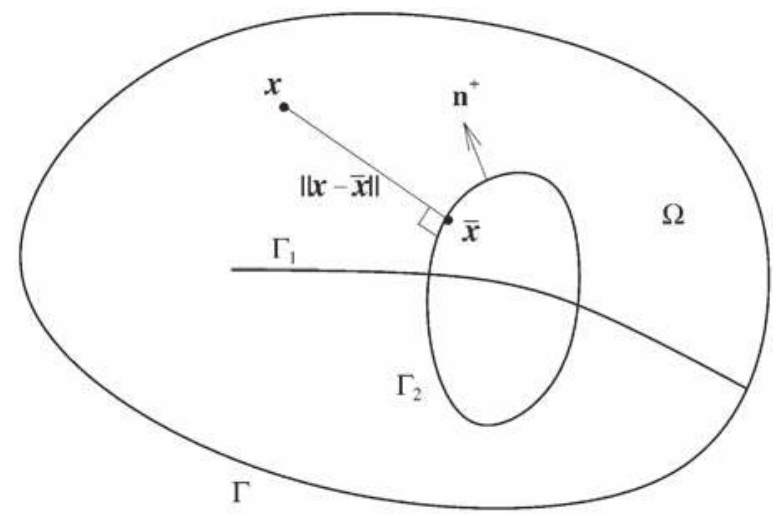

Figure 1. Illustration of nomenclature for two surfaces of discontinuity.

Other papers which address the issue of discontinuous elements are Oliver [6, 7], Armero and Garikipati [8] and Duarte et al. [9]. The methods proposed here differ from the first two in that there are no incompatibilities in the element and the discontinuities can end within an element. The method of Duarte et al. [9] provides an alternative method for discontinuous functions.

The surfaces of discontinuity are defined by signed distance functions. This description is not necessary for the application of these discontinuous approximations, but they are very appealing because the methodology of level sets can then be applied to update these surfaces for moving discontinuities, Sethian [10]. Other workers who have used level sets with finite elements are Rao et al. [11] and Stolarski et al. [12].

The major appeal of these methods for incorporating discontinuities in finite elements is that they do not require the mesh to conform to discontinuities in the approximating function or its derivatives. They also avoid remeshing for moving discontinuities. Meshing, particularly with triangles and tetrahedrons, has achieved a high level of robustness and speed, and many are tempted to use it for everything. It is our belief that in many cases, methods that avoid remeshing are preferable, for the costs of remeshing lie not only in the cost of creating a new mesh, but the tremendous overhead associated with adapting visualization techniques and other post-processing features, such as time histories of selected points, to sequences of meshes in evolution problems.

\section{APPROXIMATION FOR DISCONTINUOUS FUNCTIONS}

\subsection{Discontinuities in functions}

We consider a domain $\Omega$ with boundary $\Gamma$ as shown in Figure 1. We first describe the method for the approximation of a scalar variable $u(\mathbf{x})$ but the method is easily extended to vector fields.

The surface discontinuities in the dependent variable $u(\mathbf{x})$ are denoted by $\Gamma_{\alpha}, \alpha=1$ to $m$, where $m$ is the number of discontinuities. We first consider the construction of an approximation that is itself discontinuous on $\Gamma_{\alpha}$, which is often called a strong discontinuity. The mesh is completely independent of the geometry or location of the discontinuity. We denote 
the shape functions at node $I$ by $N_{I}(\mathbf{x})$ and the corresponding nodal values of the dependent variable by $u_{I}$.

The approximations will be of the following form:

$$
u(\mathbf{x})=\sum_{I} N_{I}(\mathbf{x})\left(u_{I}+a_{I} \phi_{I}(\mathbf{x})\right)=\sum_{I} \psi_{I}\left(\mathbf{x}, a_{I}, u_{I}\right)
$$

where $u_{I}$ are nodal values and $a_{I}$ are additional degrees of freedom associated with the enrichment $\phi_{I}(\mathbf{x})$ for the discontinuity. We will sometimes use the last form because the enrichment varies from node to node and many nodes require no enrichment. It can be seen that this is an application of the partition of unity concept [13].

Although the surfaces can be represented by any technique, for convenience we describe the surfaces of discontinuity $\Gamma_{\alpha}$ by signed distance functions $f_{\alpha}(\mathbf{x})$. The signed distance function is defined by

$$
f_{\alpha}(\mathbf{x})=\min _{\overline{\mathbf{x}} \in \Gamma_{\alpha}}\|\mathbf{x}-\overline{\mathbf{x}}\| \operatorname{sign}\left(\mathbf{n}^{+} \cdot(\overline{\mathbf{x}}-\mathbf{x})\right)
$$

where $\overline{\mathbf{x}}$ is a point on the surface of discontinuity $\Gamma_{\alpha}$ and $\mathbf{n}^{+}$is a unit normal to the surface of discontinuity from the subdomain where the distance function is positive. As is well known, the point $\overline{\mathbf{x}}$ is the closest point projection of $\mathbf{x}$ on $\Gamma_{\alpha}$, which is the orthogonal projection for a continuously differentiable surface; see Figure 1 for a depiction of the ingredients of the above equation at a typical point. We usually approximate the distance function by a finite element or meshless approximation

$$
f_{\alpha}(\mathbf{x})=\sum_{I} f_{\alpha I} N_{I}(\mathbf{x})
$$

where $N_{I}(\mathbf{x})$ are the shape functions. When $N_{I}(\mathbf{x})$ are the standard $C^{0}$ finite element shape functions, the surface (or line in 2D) of discontinuity is $C^{0}$, i.e. piecewise continuously differentiable. If smoother representations of the surface are desired, moving least square approximations such as those described for meshless methods in Reference [5] can be used, even when the solution approximation is based on finite elements. The representation of the discontinuity by Equation (3) enables it to be described completely by nodal data.

The approximation at a node $I$ depends on whether the support of $N_{I}(\mathbf{x})$ (i.e. the domain on which $N_{I}(\mathbf{x})$ is non-zero) is bisected (i.e. cut completely in two) by the discontinuity or the discontinuity ends within the support of $N_{I}(\mathbf{x})$. The two cases are illustrated in Figure 2. The support of $N_{I}(\mathbf{x})$ generally includes the domains of all elements which share node $I$. When any element in the support of $N_{I}(\mathbf{x})$ is bisected by the discontinuity, we call it a bisected support. If the discontinuity only partially cuts the support, we call it a slit support.

On a bisected support, the enrichment for representing a discontinuity in the function is given by

$$
\phi_{I}(\mathbf{x})=H\left(f_{\alpha}(\mathbf{x})\right)
$$

where $H(x)$ is a step function given by

$$
H(x)= \begin{cases}0 & \text { for } x<0 \\ +1 & \text { for } x>0\end{cases}
$$



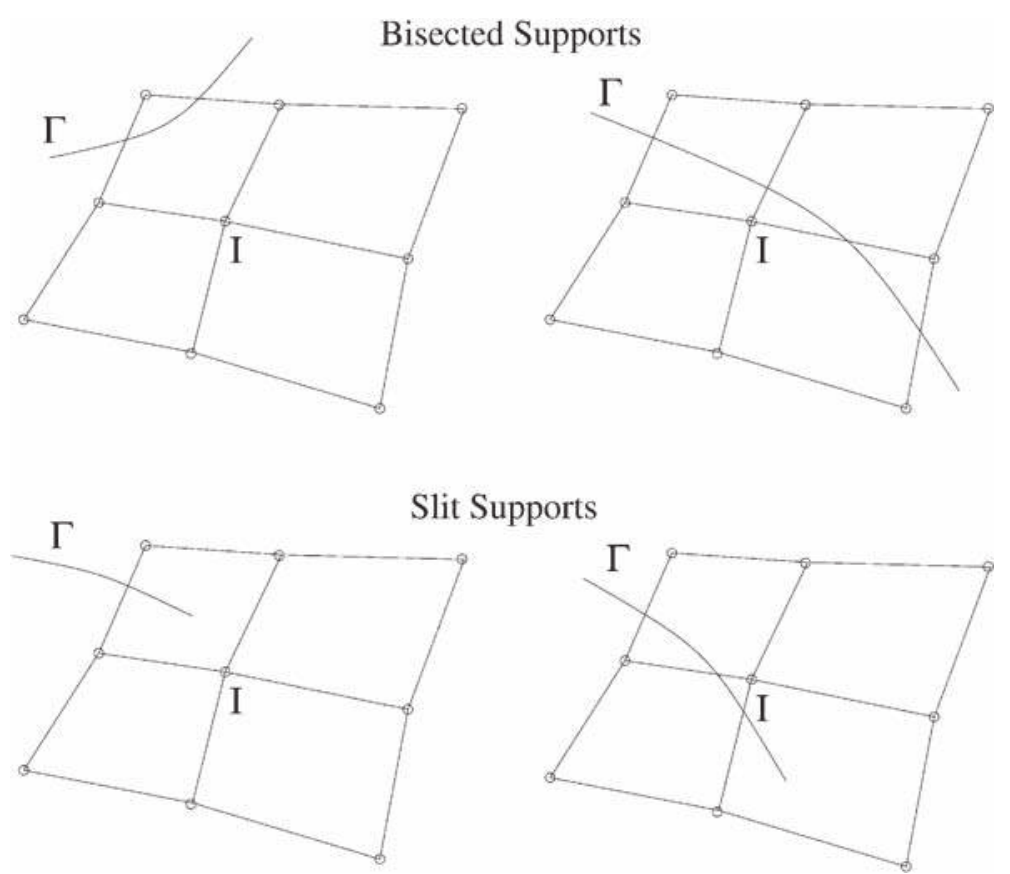

Slit Supports

Figure 2. Bisected and slit supports.

Comparing Equation (4) with Equation (1) we can see that the approximation function at an enriched node is

$$
\psi_{I}\left(\mathbf{x}, u_{I}, a_{I}\right)=N_{I}(\mathbf{x})\left(u_{I}+a_{I} H\left(f_{\alpha}(\mathbf{x})\right)\right)
$$

The above enrichment introduces the step function along the curve $f_{\alpha}(\mathbf{x})=0$. The coefficients $a_{I}$ are additional unknowns in the discrete equations and govern the magnitude of the discontinuity in the domain of the support of the shape function $N_{I}(\mathbf{x})$.

The above can be viewed as an enrichment with a windowed step function, where $N_{I}(\mathbf{x})$ is the window function. The window function localizes the enrichment so that the discrete equations will be sparse.

For a slit support, the approximation for a node is given by

$$
\psi_{I}\left(\mathbf{x}, u_{I}, a_{I}\right)=N_{I}(\mathbf{x})\left(u_{I}+\sum_{\beta} a_{I \beta} b_{\beta}(\mathbf{x})\right)
$$

where $b_{\beta}(\mathbf{x})$ are branch functions around the discontinuity. The branch functions are constructed in terms of the geometry of the surface of the discontinuity.

Consider for example the discontinuity shown in Figure 3. The virtual extension of the surface of discontinuity is constructed by

$$
\nabla f_{\alpha} \cdot\left(\mathbf{x}-\mathbf{x}_{A}\right)=0
$$

and the signed distance function is extended on the basis of this virtual extension. 


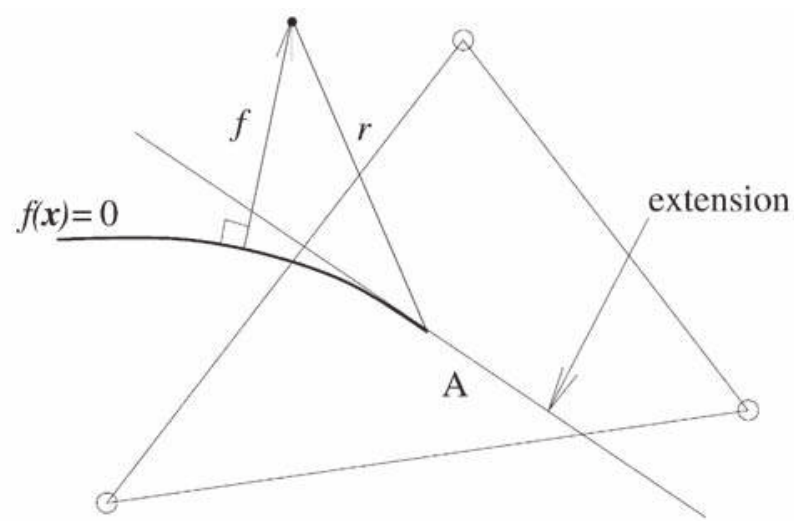

Figure 3. Construction of extension and other nomenclature for the end of a crack, point A.

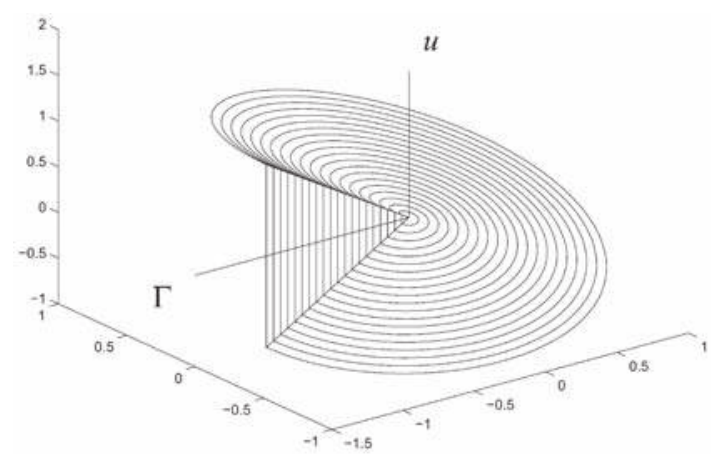

(a)

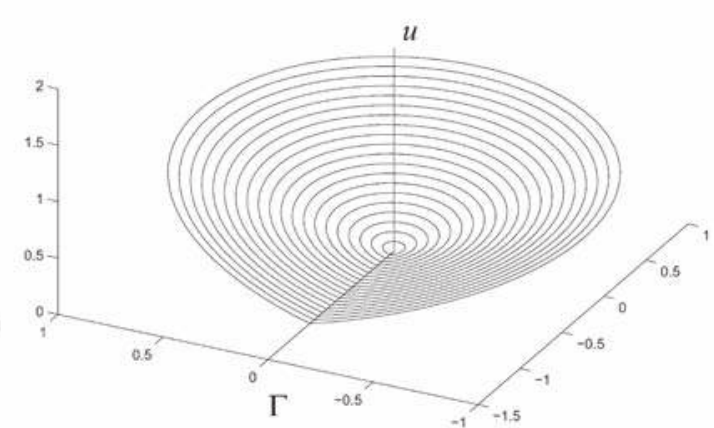

(b)

Figure 4. Branch functions for discontinuities in: (a) the function; and (b) its derivative

The branch function is constructed in terms of the distance function. The angle $\theta$ for any point $\mathbf{x}$ is defined by

$$
\theta(\mathbf{x})=\tan ^{-1}\left(\frac{f(\mathbf{x})}{r}\right), \quad-\pi<\theta<\pi
$$

This construction enables the discontinuity to be curved or piecewise linear, since by defining the angle in terms of $f(\mathbf{x})$ the line of discontinuity always corresponds to $f(\mathbf{x})=0$.

The branch function is then constructed in terms of the radial co-ordinate of point $\mathrm{A}$ and $\theta$; see Figure 3. Generally more than one branch function is needed for each enriched node. For example, the enrichment functions can be

$$
b_{i}(\mathbf{x})=\left[r \sin \frac{\theta}{2}, r^{2} \sin \frac{\theta}{2}\right]
$$

The linear branch function is illustrated in Figure 4(a). As can be seen, the function is discontinuous across the line $\Gamma_{\alpha}$ and is continuous and well behaved in the domain surrounding the discontinuity. For linear fracture mechanics, the functions

$$
b_{i}(\mathbf{x})=\left[\sqrt{r} \sin \frac{\theta}{2}, \sqrt{r} \sin \frac{\theta}{2} \sin \theta, \sqrt{r} \cos \frac{\theta}{2}, \sqrt{r} \cos \frac{\theta}{2} \sin \theta\right]
$$




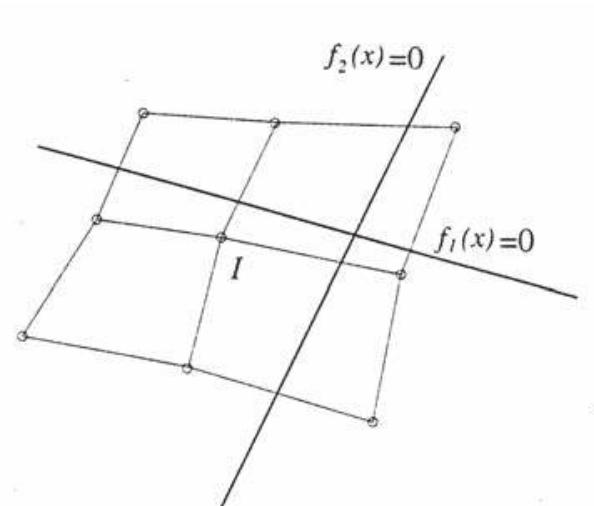

(a)

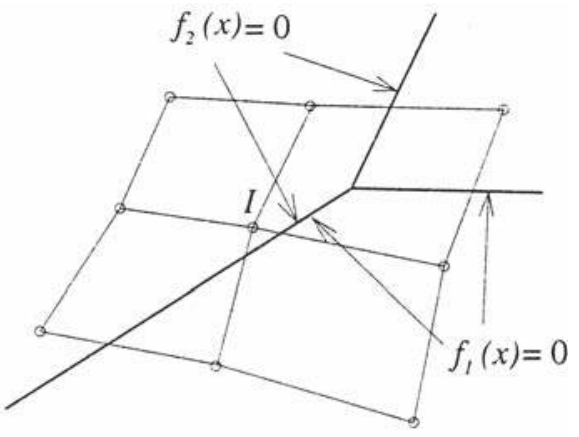

(b)

Figure 5. Support of node I with: (a) intersecting discontinuities; and (b) branching discontinuities

have been used in Reference [1]. Only the first of the above functions is a branch function; the others were added to improve the accuracy in elastic fracture problems. For the same reason, the enrichment was chosen to vary with $\sqrt{r}$. The above functions span the near-tip asymptotic solution for a crack, so very good accuracy can be obtained for these problems.

\subsection{Intersecting discontinuities}

For nodes whose supports are cut by two or more discontinuities, the enrichment must include additional functions as shown in Daux et al [3]. Consider for example the case of two discontinuities which intersect in a support as shown in Figure 5(a). The approximation function for the discontinuity is then given by

$$
\psi_{I}\left(\mathbf{x}, u_{I}, a_{I}\right)=N_{I}(\mathbf{x})\left(u_{I}+a_{I 1} H\left(f_{1}(\mathbf{x})\right)+a_{I 2} H\left(f_{2}(\mathbf{x})\right)+a_{I 3} H\left(f_{1}(\mathbf{x}) f_{2}(\mathbf{x})\right)\right.
$$

Thus we need three additional unknowns at each node for which the support contains the intersection of the two discontinuities. These functions are linearly independent and linearly independent of the finite element shape functions. The above form is somewhat different from Daux et al. [3], but more compact and more easily implemented.

Another important case is a branching discontinuity, such as a branching crack shown in Figure 5(b). This is a degeneration of the above case of intersecting discontinuities. The approximation function for the nodes whose support contains the branch is

$$
\psi_{I}\left(\mathbf{x}, u_{I}, a_{I}\right)=N_{I}(\mathbf{x})\left(u_{I}+a_{I 1} H\left(f_{1}(\mathbf{x})\right)+a_{I 2} H\left(f_{2}(\mathbf{x})\right)\right)
$$

The enrichment here consists of two linearly independent functions. It is also possible to add enrichments to account for the singularities which occur at intersecting discontinuities in elastic materials.

\subsection{Discontinuities in derivatives}

Another important class of discontinuities are those in the first derivatives of the approximation. These discontinuities occur at interfaces between materials and different phases of materials, such as at an austenite-martensite interface. Discontinuities in derivatives of solutions 
occur wherever the coefficients of the governing partial differential equation are discontinuous. These discontinuities can easily be handled by standard finite element approximations by aligning the element edges with the discontinuity. However, if the discontinuity moves with time, remeshing is required. The approximation given below can model discontinuities in the derivatives on surfaces or (lines in two dimensions) which are independent of the mesh. An approximation with a discontinuity in the derivative is constructed by letting the nodal approximation for a node with a bisected support be

$$
\psi_{I}\left(\mathbf{x}, u_{I}, a_{I}\right)=N_{I}(\mathbf{x})\left(u_{I}+a_{I}\left|f_{\alpha}(\mathbf{x})\right|\right)
$$

where $|\cdot|$ is the absolute value symbol. The enrichment is thus the absolute value of the signed distance function. This concept was introduced in Reference [14].

For a support which is slit by the discontinuity, the approximation is

$$
\psi_{I}\left(\mathbf{x}, u_{I}, a_{I}\right)=N_{I}(\mathbf{x})\left(u_{I}+\sum_{\alpha} a_{I \alpha} b_{I \alpha}^{\mathrm{D}}\left(\mathbf{x}_{I}\right)\right)
$$

where $b_{I \alpha}^{\mathrm{D}}\left(\mathbf{x}_{I}\right)$ are branch functions such as

$$
b_{I \alpha}^{\mathrm{D}}\left(\mathbf{x}_{I}\right)=\left[r \cos \frac{\theta}{2}, r^{2} \cos \frac{\theta}{2}, \sqrt{r} \cos \frac{\theta}{2}\right]
$$

The first branch function in (16) is illustrated in Figure 4(b). As can be seen, it is continuously differentiable within the domain but is cusplike across $\Gamma$.

For intersecting discontinuities in derivatives, the same procedure as for discontinuities in the function is used. For example, for the nodes whose support contain two intersecting discontinuities, the approximation is

$$
\psi_{I}\left(\mathbf{x}, u_{I}, a_{I}\right)=N_{I}(\mathbf{x})\left(u_{I}+a_{I 1}\left|f_{1}(\mathbf{x})\right|+a_{I 2}\left|f_{2}(\mathbf{x})\right|+a_{I 3}\left|f_{1}(\mathbf{x})\right|\left|f_{2}(\mathbf{x})\right|\right)
$$

Discontinuous branching derivatives can be constructed by adapting (13):

$$
\psi_{I}\left(\mathbf{x}, u_{I}, a_{I}\right)=N_{I}(\mathbf{x})\left(u_{I}+a_{I 1}\left|f_{1}(\mathbf{x})\right|+a_{I 2}\left|f_{2}(\mathbf{x})\right|\right)
$$

\subsection{Vector functions and tangential discontinuities}

The above can easily be generalized to vector functions. For example, for a vector function $\mathbf{u}(\mathbf{x})$, such as a displacement, a discontinuity on $f(\mathbf{x})$ is introduced by

$$
\mathbf{u}(\mathbf{x})=\sum_{I} N_{I}\left(\mathbf{u}_{I}+\mathbf{a}_{I} H(f(\mathbf{x}))\right)
$$

where $\mathbf{a}_{I}$ is a column matrix of the same dimension as $\mathbf{u}_{I}$.

The construction of discontinuities of a single component in a vector function is simplified by the use of the signed distance function. This approach can be used to model shear bands and cracks which have closed due to compressive forces where the tangential displacement is discontinuous. The unit normal to the line of discontinuity is given by

$$
\mathbf{e}_{n}=\frac{\nabla f}{\|\nabla f\|}
$$

Although a signed distance function should have a unit gradient, we normalize it here since this should be done in a computation. The tangent plane is then defined by any two unit vectors orthogonal to $\mathbf{e}_{n}$. 
We illustrate the construction of the approximation in two dimensions. The discontinuity in the tangential component is obtained by letting the displacement field in the elements cut by the discontinuity be given by

$$
\mathbf{u}(\mathbf{x})=\sum_{I} N_{I}(\mathbf{x})\left(\mathbf{u}_{I}+a_{I} \mathbf{e}_{t}(\mathbf{x}) H(f(\mathbf{x}))\right)
$$

where $\mathbf{e}_{t}=\mathbf{e}_{z} \times \mathbf{e}_{n}$ is a vector in the tangent direction. Only a single parameter is needed at each node.

\subsection{Comparison to Global-Local}

The above methods can be viewed as generalizations of the well-known global-local methods. In the partition of unity method, the global approximation is multiplied by a window function to enhance the approximation properties of the global function and to improve the sparsity of the discrete equations. If we consider a standard global-local approximation with a global function $g(\mathbf{x})$, then the approximation of $u(\mathbf{x})$ is

$$
u(\mathbf{x})=\operatorname{ag}(\mathbf{x})+\sum N_{I}(\mathbf{x}) u_{I}
$$

Compare with the partition of unity approximation

$$
u(\mathbf{x})=\sum_{I} N_{I}(\mathbf{x})\left(u_{I}+a_{I} g(\mathbf{x})\right)
$$

It can be seen that in this method, the global function $g(\mathbf{x})$ can adjust itself within the domain. In addition, the function $g(\mathbf{x})$ can be introduced only in the subdomain where it is needed. The structure of the approximation yields a smooth function even when $a_{I}$ are chosen to vanish at certain nodes.

Comparing (23) to (22), we see the following key differences

1. In the classical global-local approximation the global function is indeed global so that the equations are not sparse. On the other hand, in (23) the global function $g(\mathbf{x})$ is multiplied by a window which localizes it, so that the discrete equations are sparse.

2. The parameters $a_{I}$ in (23) enable the approximation better fit the particular solution at hand, since they can vary from node to node; on the other hand, they also introduce additional unknowns into the system.

3. In the partition of unity, the enrichment can be added only where it is needed.

\section{WEAK FORM FOR LAPLACE EQUATION}

In order to introduce the weak form for functions with interior discontinuities in a simple setting, we first consider the Laplace equation with discontinuous coefficients over a domain $\Omega$ and boundary $\Gamma$. The strong form is

$$
\begin{aligned}
\nabla \cdot(\alpha(\mathbf{x}) \nabla u(\mathbf{x}))=0 & \text { on } \Omega \\
\llbracket \alpha(\mathbf{x}) u(\mathbf{x}),_{N} \rrbracket \equiv \alpha^{+} \nabla u^{+} \cdot \mathbf{n}^{+}+\alpha^{-} \nabla u^{-} \cdot \mathbf{n}^{-}=0 & \text { on } \Gamma_{\mathrm{D}}
\end{aligned}
$$




$$
\begin{aligned}
u(\mathbf{x})_{,_{N}}=\nabla u(\mathbf{x}) \cdot \mathbf{n} & =0 & & \text { on } \Gamma_{F}^{+} \text {and } \Gamma_{F}^{-} \\
\alpha(\mathbf{x}) u(\mathbf{x})_{N}=\alpha(\mathbf{x}) \nabla u(\mathbf{x}) \cdot \mathbf{n} & =h & & \text { on } \Gamma_{h} \\
u & =g & & \text { on } \Gamma_{g}
\end{aligned}
$$

where $\mathbf{n}$ is the unit normal to the surface, the superscripts ' + ' and ' - ' designate two sides of the interface and $\alpha(\mathbf{x})$ is a $C^{-1}$ function. The solution is discontinuous on $\Gamma_{F}$ and its derivatives are discontinuous on $\Gamma_{\mathrm{D}}$. Note that in a finite element application with $C^{0}$ shape functions, $\Gamma_{\mathrm{D}}$ includes all element interfaces.

The space of trial and test functions are

$$
\begin{aligned}
\mathscr{U} & =\left\{u(\mathbf{x}) \mid u(\mathbf{x}) \in \mathscr{H}_{1}, u(\mathbf{x})=g \text { on } \Gamma_{u}, u(\mathbf{x}) \text { discontinuous on } \Gamma_{F}\right\} \\
\mathscr{U}_{0} & =\left\{\delta u(\mathbf{x}) \mid \delta u(\mathbf{x}) \in \mathscr{H}_{1}, \delta u(\mathbf{x})=0 \text { on } \Gamma_{u}, \delta u(\mathbf{x}) \text { discontinuous on } \Gamma_{F}\right\}
\end{aligned}
$$

The weak form is: find $u \in \mathscr{U}$, such that

$$
\int_{\Omega} \nabla \delta u \cdot \alpha \nabla u \mathrm{~d} \Omega-\int_{\Gamma_{h}} \delta u h \mathrm{~d} \Gamma=0 \quad \forall \delta u \in \mathscr{U}_{0}
$$

We next show that the above implies Equation (24) and all interface and natural (Neumann) boundary conditions. The first term on the left-hand side of the above can be integrated by parts to give

$$
\int_{\Omega} \nabla \delta u \cdot \alpha \nabla u \mathrm{~d} \Omega=\int_{\Omega}(\nabla(\delta u \alpha \nabla u)-\delta u \nabla \cdot(\alpha \nabla u)) \mathrm{d} \Omega
$$

By Gauss's theorem, the first term on the right-hand side of the above gives

$$
\int_{\Omega} \nabla(\delta u \alpha \nabla u) \mathrm{d} \Omega=\int_{\Gamma_{h}}(\delta u \alpha \nabla u \cdot \mathbf{n}) \mathrm{d} \Gamma+\int_{\Gamma_{F} \cup \Gamma_{\mathrm{D}}}\left(\delta u^{+} \alpha^{+} \nabla u^{+} \cdot \mathbf{n}^{+}+\delta u^{-} \alpha^{-} \nabla u^{-} \cdot \mathbf{n}^{-}\right) \mathrm{d} \Gamma
$$

We now note that by the definition of the test space, $\delta u^{+}=\delta u^{-}$on $\Gamma_{\mathrm{D}}$ so the integrand of the second term over $\Gamma_{\mathrm{D}}$ becomes $\delta u \llbracket \alpha u, N \rrbracket$. Substituting Equation (33) into Equation (32) and in turn into Equation (31) gives

$$
\begin{gathered}
-\int_{\Omega} \delta u \nabla \cdot(\alpha \nabla u) \mathrm{d} \Omega+\int_{\Gamma_{h}} \delta u\left(\alpha u,_{N}-h\right) \mathrm{d} \Gamma+\int_{\Gamma_{\mathrm{D}}} \delta u \llbracket \alpha u,{ }_{N} \rrbracket \mathrm{d} \Gamma \\
\int_{\Gamma_{F}}\left(\delta u^{+} \alpha^{+} \nabla u^{+} \cdot \mathbf{n}^{+}+\delta u^{-} \alpha^{-} \nabla u^{-} \cdot \mathbf{n}^{-}\right) \mathrm{d} \Gamma=0
\end{gathered}
$$

Equations (24), (25) and (27) follow immediately from the first three terms of the above by the density theorem. Since $\delta u$ is discontinuous on $\Gamma_{F}, \delta u^{+}$and $\delta u^{-}$are arbitrary, and it follows 

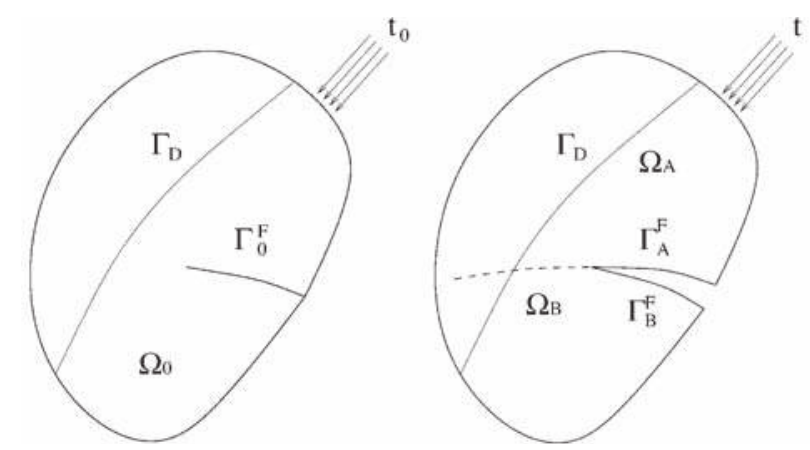

Figure 6. Initial and current configuration showing the splitting of $\Gamma_{F}^{0}$.

that

$$
\begin{array}{ll}
\nabla u^{+} \cdot \mathbf{n}^{+}=u,{ }_{N}^{+}=0 & \text { on } \Gamma_{F} \\
\nabla u^{-} \cdot \mathbf{n}^{-}=u,{ }_{N}=0 & \text { on } \Gamma_{F}
\end{array}
$$

So Equations (24)-(28) in the strong form are implied by the weak form (31). Therefore the approximations with interior discontinuities described in the previous section are applicable to the solution of this class of problems.

\section{WEAK FORM FOR DISCONTINUOUS MOTION}

In the following we give the weak form for the equilibrium equation of solid mechanics when the motion has discontinuities and discontinuous derivatives. We will show that the strong form, in addition to the equilibrium equation and traction boundary conditions, includes the appropriate jump conditions at the interfaces. We consider a body $\Omega$ with reference configuration $\Omega_{0}$. The body is subjected to body forces $\rho_{0} \mathbf{b}$ per unit volume and tractions $\mathbf{t}_{0}$ on the boundary of the reference configuration. We denote the nominal stress by $\mathbf{P}$ and the deformation gradient by $\mathbf{F}, F_{i j}=\partial x_{i} / \partial X_{j}$. The motion is described by a map

$$
\mathbf{x}=\boldsymbol{\Phi}(\mathbf{X}, t)
$$

This map must be one-to-one and onto, i.e. bijunctive, and continuously differentiable everywhere except on a finite number of sets of measure zero, where the motion or its derivatives may be discontinuous. The discontinuities in derivatives correspond to entities such as material interfaces. The discontinuities in the motion correspond to entities such as cracks and shear bands. The motion on the surfaces of discontinuity is interesting, since a surfaces splits into two surfaces. This is illustrated in Figure 6 which shows the initial and current configurations of a body which has cracked. As can be seen, the surface from which the crack emanates splits into two surfaces, so any point on the reference surface becomes two points. In the reference configuration, the crack always remains two coincident surfaces whereas in the current configuration the crack becomes two surfaces separated by a gap. Thus the map for points on the surface is not one-to-one. In fact, the points on the surface split into two points. 
Jumps in the derivatives of the motion occur on surfaces $\Gamma_{0}^{\mathrm{D}}$ and the motion is discontinuous on surfaces $\Gamma_{0}^{F}$; for simplicity of notation we restrict this treatment to a single surface. The surface $\Gamma_{0}^{F}$ then splits into two surfaces $\Gamma_{\mathrm{A}}^{F}$ and $\Gamma_{\mathrm{B}}^{F}$. The motion of points on $\Gamma_{0}^{F}$ is subject to rather complex conditions which stem from the fact that across the surface $\Gamma_{0}^{F}$ the behaviour is identical to that of two bodies in contact. Therefore, once the discontinuity has developed, interpenetration must be prevented, i.e. we must attach conditions on the motion so that the impenetrability conditions are met, see Belytschko et al. [15]. To facilitate the expression of this condition, we subdivide the body into two by extending the surface $\Gamma_{F}^{0}$ until the body is bisected. The impenetrability conditions are

$$
\text { if } \mathbf{X} \in \Gamma_{0 \mathrm{~A}}^{F} \quad \text { then } \mathbf{x}(\mathbf{X}, t) \notin \Omega_{\mathrm{B}}, \quad \text { if } \mathbf{X} \in \Gamma_{0 \mathrm{~B}}^{F} \quad \text { then } \mathbf{x}(\mathbf{X}, t) \notin \Omega_{\mathrm{A}}
$$

The above state that any point on one side of a crack cannot interpenetrate the other side. Once the crack develops, the surface $\Gamma_{0}^{F}$ changes character. If the surface splits, i.e. if the crack opens, then $\Gamma$ becomes two traction surfaces.

An interesting feature of this motion is that the surfaces $\Gamma_{0}^{F}$ are not known in the undeformed body. The surfaces develop as the deformation proceeds, and their description in terms of the reference configuration has to be made by an inverse map of the motion. Thus if the discontinuity at time $\tau, \Gamma^{F}(\tau)$, is described by $f(\mathbf{x}, \tau)=0$, then the surface of discontinuity in the undeformed configuration $\Gamma_{0}^{F}$ is given by $f\left(\Phi^{-1}(\mathbf{x}, \tau), \tau\right)=0$. The preceding function is difficult to construct, but is in fact never needed; the surface of discontinuity $\Gamma_{0}^{F}$ in the reference configuration is a conceptual device that is never invoked in actual computations.

The conditions in (38) are difficult to enforce directly, so we will deal with the cracks in which the shear is small, so we assume that points which contact were originally contiguous. The gap is given by

$$
g_{N} \equiv \llbracket \mathbf{u}, N \rrbracket=\mathbf{u}^{\mathrm{A}} \cdot \mathbf{n}^{\mathrm{A}}+\mathbf{u}^{\mathrm{B}} \cdot \mathbf{n}^{\mathrm{B}} \leqslant 0
$$

This expression is approximate and only applies if interpenetration only occurs for points that are initially contiguous. We assume the crack is frictionless when in contact. The strong form consists of the following:

$$
\begin{array}{rlrl}
\frac{\partial P_{j i}}{\partial X_{j}}+\rho_{0} b_{i} & =0 & \text { in } \Omega_{0} \\
n_{j}^{0} P_{j i} & =\bar{t}_{i} & & \\
n_{j}^{0} P_{j i} & =0 & & \text { on } \Gamma_{0}^{\mathrm{t}} \\
\hat{t}_{\alpha}^{0 \mathrm{~A}}=\hat{t}_{\alpha}^{0 \mathrm{~B}}=0, \quad \text { if not in contact } \\
t_{N}^{0 \mathrm{~A}}+t_{N}^{0 \mathrm{~B}} & =0 & & \text { on } \Gamma_{0}^{F} \quad \text { if in contact } \\
\llbracket n_{j}^{0} P_{j i} \rrbracket & =0 & \text { on } \Gamma_{0}^{\mathrm{D}} & \\
\llbracket \mathbf{u}_{N} \rrbracket & \leqslant 0 & & \text { on } \Gamma_{0}^{F} \\
u_{i} & =\bar{u}_{i} & & \text { on } \Gamma_{0}^{u}
\end{array}
$$

where $t_{N}^{0}=n_{j}^{0} P_{j i} n_{j}^{0}$ and $\hat{t}_{\alpha}^{0}$ are the tangential tractions. We have assumed that the crack surfaces which are generated are traction free. 
The trial functions reside in the following space:

$$
\mathscr{U}=\left\{\mathbf{u}(\mathbf{X}, t) \mid \mathbf{u}(\mathbf{X}, t) \in C^{0}, \mathbf{u}(\mathbf{X}, t)=\overline{\mathbf{u}}(t) \text { on } \Gamma_{0}^{u}, \mathbf{u} \text { discontinuous on } \Gamma_{0}^{F}\right\}, \quad \lambda \geqslant 0, \lambda \in C^{-1}
$$

The space of test functions is defined by

$$
\mathscr{U}_{0}=\left\{\delta \mathbf{u}(\mathbf{X}) \mid \delta \mathbf{u}(\mathbf{X}) \in C^{0}, \delta \mathbf{u}(\mathbf{X})=0 \text { on } \Gamma_{0}^{u}, \delta \mathbf{u} \text { discontinuous on } \Gamma_{0}^{F}\right\}, \quad \delta \lambda \geqslant 0, \delta \lambda \in C^{-1}
$$

The contact conditions will be imposed by Lagrange multipliers. The weak form is an inequality: find $\mathbf{u}(\mathbf{X}, t) \in \mathscr{U}$ and $\lambda(\mathbf{X}, t) \in C^{-1}$ so that

$$
\int_{\Omega_{0}}\left(\delta \mathbf{F}^{\mathrm{T}}: \mathbf{P}-\rho_{0} \delta \mathbf{u} \cdot \mathbf{b}\right) \mathrm{d} \Omega_{0}-\int_{\Gamma_{0}^{\mathrm{t}}} \delta \mathbf{u} \cdot \overline{\mathbf{t}}_{0} \mathrm{~d} \Gamma_{0}+\delta \int_{\Gamma_{0}^{F}} \lambda \llbracket \mathbf{u}_{N} \rrbracket \mathrm{d} \Gamma_{0} \geqslant 0
$$

Next, we deduce the strong form from the weak form. Writing the first term in indicial form and integrating by parts gives

$$
\int_{\Omega_{0}} \frac{\partial\left(\delta u_{i}\right)}{\partial X_{j}} P_{j i} \mathrm{~d} \Omega_{0}=\int_{\Gamma_{0}^{\mathrm{t}}} \delta u_{i} n_{j}^{0} P_{j i} \mathrm{~d} \Gamma_{0}+\int_{\Gamma_{0}^{F} \cup \Gamma_{0}^{\mathrm{D}}}\left(\delta u_{i}^{\mathrm{A}} n_{j}^{0 \mathrm{~A}} P_{j i}^{\mathrm{A}}+\delta u_{i}^{\mathrm{B}} n_{j}^{0 \mathrm{~B}} P_{j i}^{\mathrm{B}}\right) \mathrm{d} \Gamma_{0}-\int_{\Omega_{0}} \delta u_{i} \frac{\partial P_{j i}}{\partial X_{j}} \mathrm{~d} \Omega_{0}
$$

We now expand the surface terms in normal and tangential components, which gives for each term the following

$$
\int_{\Gamma_{0}^{F}} \delta u_{i}^{\mathrm{A}} n_{j}^{0 \mathrm{~A}} P_{j i} \mathrm{~d} \Gamma_{0}=\int_{\Gamma_{0}^{F}}\left(\delta u_{N}^{\mathrm{A}} t_{N}^{0 \mathrm{~A}}+\delta \hat{u}_{\alpha}^{\mathrm{A}} \hat{t}_{\alpha}^{0 \mathrm{~A}}\right) \mathrm{d} \Gamma_{0}
$$

Substituting Equation (51) into Equation (50), then into Equation (49) and collecting terms gives

$$
\begin{aligned}
& \int_{\Omega_{0}} \delta u_{i}\left(-\frac{\partial P_{j i}}{\partial X_{j}}-\rho_{0} b_{i}\right) \mathrm{d} \Omega_{0}+\int_{\Gamma_{0}^{\mathrm{t}}} \delta u_{i}\left(n_{j}^{0} P_{j i}-\bar{t}_{j}^{0}\right) \mathrm{d} \Gamma_{0} \\
& +\int_{\Gamma_{0}^{F} \cup \Gamma_{0}^{\mathrm{D}}}\left(\delta u_{N}^{\mathrm{A}} t_{N}^{0 \mathrm{~A}}+\delta u_{N}^{\mathrm{B}} t_{N}^{0 \mathrm{~B}}+\delta \hat{u}_{\alpha}^{\mathrm{A}} \hat{t}_{\alpha}^{0 \mathrm{~A}}+\delta \hat{u}_{\alpha}^{\mathrm{B}} \hat{t}_{\alpha}^{0 \mathrm{~B}}\right) \mathrm{d} \Omega_{0} \\
& +\int_{\Gamma_{0}^{F}}\left(\delta \lambda \llbracket u_{N} \rrbracket+\lambda\left(\delta u_{N}^{\mathrm{A}}-\delta u_{N}^{\mathrm{B}}\right)\right) \mathrm{d} \Gamma_{0} \geqslant 0
\end{aligned}
$$

Since the above holds for all $\delta \mathbf{u} \in \mathscr{U}_{0}$, it follows by the density theorem from the first two terms of the above that the equilibrium equation (40) holds on $\Omega_{0}$ and the traction boundary conditions (41) hold on $\Gamma_{0}^{\mathrm{t}}$. 
On $\Gamma_{0}^{F}, \delta u_{N}$ is arbitrary, so

$$
t_{N}^{0 \mathrm{~A}}=-\lambda, \quad t_{N}^{0 \mathrm{~B}}=\lambda
$$

and combining the above gives

$$
t_{N}^{0 \mathrm{~A}}+t_{N}^{0 \mathrm{~B}}=0 \quad \text { on } \Gamma_{0}^{F}
$$

Since $\delta \lambda \leqslant 0$ it follows from Equation (52) that

$$
\llbracket u_{N} \rrbracket \leqslant 0 \quad \text { on } \Gamma_{0}^{F}
$$

On $\Gamma_{0}^{\mathrm{D}}, \delta \mathbf{u}^{\mathrm{A}}=\delta \mathbf{u}^{\mathrm{B}}$ so it follows that

$$
t_{i}^{0 \mathrm{~A}}=t_{i}^{0 \mathrm{~B}} \quad \text { or } \quad \llbracket n_{j}^{0} P_{j i} \rrbracket=0
$$

\section{DISCRETIZATION}

We will describe the discretization for the case where discontinuities in the tangential displacements are small enough so that we can assume any contact occurs on originally contiguous material points. This assumption is valid for most cracks. It is also applicable to the shear band models described before if the relative displacements are small. If large shears occur across a line of discontinuity, then any recontact may occur on material points which were not originally contiguous, and general algorithms for contact must be used. However, for many technologically important problems in fracture, the simpler formulation given here is applicable. The discretization of all but the last term is standard for the interpolations given here, so we focus on the last term. We parameterize the surface of discontinuity by $\xi^{\alpha}$, where $\alpha$ has a range of one and two for one and two-dimensional problems, respectively. We approximate the Lagrange multipliers by

$$
\lambda\left(\xi^{\alpha}, t\right)=\lambda_{I}(t) N_{I}\left(\xi^{\alpha}\right)
$$

The interpenetration function is given by

$$
\llbracket u_{N} \rrbracket=\left(u_{i}^{\mathrm{A}}-u_{i}^{\mathrm{B}}\right) n_{i}^{\mathrm{A}}=n_{i}^{\mathrm{A}} N_{I} a_{i I}\left(H\left(f\left(\mathbf{x}^{\mathrm{A}}\right)\right)-H\left(f\left(\mathbf{x}^{\mathrm{B}}\right)\right)\right)=n_{i}^{\mathrm{A}} N_{I} a_{i I}
$$

Thus the interpenetration depends strictly on the enrichment parameters. Substituting (57) and (58) into the last term in the weak form, which we will call $\delta W_{c}$, gives the following:

$$
\delta W_{c}=\int_{\Gamma_{0}^{F}} \delta\left(\lambda \llbracket u_{N} \rrbracket\right) \mathrm{d} \Gamma_{0}=\delta \lambda_{J} G_{J i I} a_{i I}+\lambda_{J} G_{J i I} \delta a_{i I}
$$

where

$$
G_{J i I}=\int_{\Gamma_{0}^{c}} n_{i}^{\mathrm{A}} N_{J}^{\lambda} N_{I} \mathrm{~d} \Gamma
$$

The discrete weak form can then be written as

$$
\delta W=\delta u_{i I}\left(f_{i I}^{\mathrm{int}}-f_{i I}^{\mathrm{ext}}\right)+\delta a_{i I}\left(q_{i I}^{\mathrm{int}}-q_{i I}^{\mathrm{ext}}\right)+\delta \lambda_{J} G_{J i I} a_{i I}+\lambda_{J} G_{J i I} \delta a_{i I}
$$


The discrete equations are

$$
\begin{aligned}
f_{i I}^{\text {int }}-f_{i I}^{\text {ext }} & =0 \\
q_{i I}^{\text {int }}-q_{i I}^{\text {ext }}+G_{J i I} \lambda_{J} & =0 \\
G_{J i} a_{i I} & \leqslant 0 \\
\lambda_{J} & \geqslant 0
\end{aligned}
$$

where in the last equation it has been assumed that the condition $\lambda_{J} \geqslant 0$ suffices to enforce $\lambda\left(\xi^{\alpha}\right) \geqslant 0$; this is true only for low-order elements. The above equations are, respectively, the equilibrium equation associated with the regular nodal displacements, the equilibrium equations for the enrichment degrees of freedom and the impenetrability constraint on the discontinuity surface. The third term in Equation (63) are the contact forces. Note that the contact forces are associated only with the enrichment. The equilibrium equations are coupled since $f^{\text {int }}$ depends on $\mathbf{u}_{I}$ and $\mathbf{a}_{I}$.

For small-displacement elastostatic problems, the discrete equations obtained from (62)(64) are

$$
\left[\begin{array}{ccc}
\mathbf{K}_{u u} & \mathbf{K}_{u a} & \mathbf{0} \\
\mathbf{K}_{u a}^{\mathrm{T}} & \mathbf{K}_{a a} & \mathbf{G}^{\mathrm{T}} \\
\mathbf{0} & \mathbf{G} & \mathbf{0}
\end{array}\right]\left\{\begin{array}{l}
\mathbf{u} \\
\mathbf{a} \\
\lambda
\end{array}\right\}=\left\{\begin{array}{c}
\mathbf{f}^{\mathrm{ext}} \\
\mathbf{q}^{\mathrm{ext}} \\
\mathbf{0}
\end{array}\right\}
$$

where $\mathbf{f}^{\text {ext }}$ and $\mathbf{q}^{\text {ext }}$ are the nodal external forces and

$$
\mathbf{K}_{u u}=\int_{\Omega} \mathbf{B}_{u}^{\mathrm{T}} \mathbf{C} \mathbf{B}_{u} \mathrm{~d} \Omega, \quad \mathbf{K}_{u a}=\int_{\Omega} \mathbf{B}_{u}^{\mathrm{T}} \mathbf{C} \mathbf{B}_{a} \mathrm{~d} \Omega, \quad \mathbf{K}_{a a}=\int_{\Omega} \mathbf{B}_{a}^{\mathrm{T}} \mathbf{C} \mathbf{B}_{a} \mathrm{~d} \Omega
$$

In the above, $\mathbf{C}$ is the standard elastic coefficient matrix; in two dimensions $\mathbf{B}_{u}$ and $\mathbf{B}_{a}$ consist of the nodal submatrices

$$
\mathbf{B}_{u I}=\left[\begin{array}{cc}
N_{I, x} & 0 \\
0 & N_{I, y} \\
N_{I, y} & N_{I, x}
\end{array}\right], \quad \mathbf{B}_{a I}=\left[\begin{array}{cc}
\phi_{I, x} & 0 \\
0 & \phi_{I, y} \\
\phi_{I, y} & \phi_{I, x}
\end{array}\right]
$$

At first glance, a major disadvantage of this methodology is that it requires a finite element system with a variable number of degrees of freedom per node. The difficulty is not as burdensome as it seems at first. The variable number of degrees of freedom can be handled by adding extra nodes to the element on an interface to handle the enrichment degrees of freedom $a_{i I}$. Thus a 3-node triangle becomes a 6-node triangle for an element bisected by a discontinuity, with the pair of nodes superimposed. The elements with enrichment degrees of freedom are then assigned to the additional nodes.

In the implementation of the discontinuous functions, it is beneficial to replace the enrichment function in Equation (4) by $\phi_{I}(\mathbf{x})=H\left(f_{\alpha}(\mathbf{x})\right)-H\left(f_{\alpha}\left(\mathbf{x}_{I}\right)\right)$. The enrichment function then vanishes in all elements except those that contain the discontinuity. 


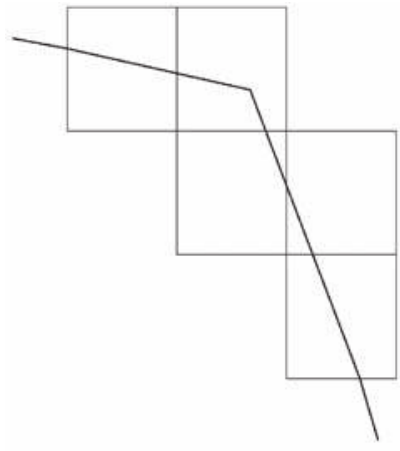

(a)

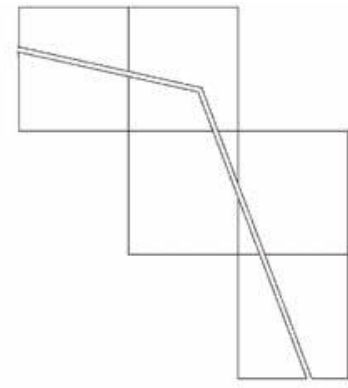

(b)

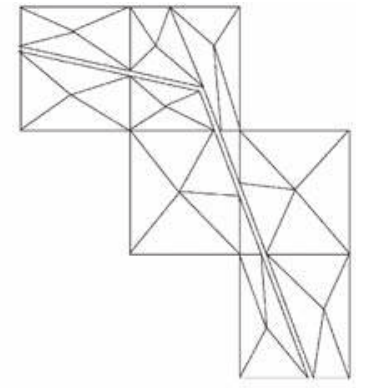

(c)

Figure 7. Generation of subpolygons for the quadrature of the weak form in: (a) elements cut by a crack. The polygons; (b) formed from the intersection of the crack and the element geometries are; (c) triangulated to create the element subdomains.

Quadrature of the weak form in elements cut by the discontinuity also requires revision from a standard program. There are three approaches to quadrature in elements cut by discontinuities:

1. A quadrature rule which does not rely highly on continuity of the integrand may be used. For example, the trapezoidal rule may be used with $5 \times 5$ quadrature points over the domain. Although this leads to moderate errors, for solutions of engineering accuracy, these methods suffice.

2. The elements which are cut by discontinuities are subdivided into subelements which do not cross the discontinuity and the element quadrature is performed over these subelements. A description of such methods may be found in Moës et al. [2], as shown in Figure 7. This approach gives higher accuracy but it involves greater programming effort.

3. A method based on adaptive subdomains as described by Strouboulis et al. [16].

In addition, it is necessary to carefully treat elements where the cut leaves a small subelement. If an enrichment is created for that case, the additional functions may be linearly dependent. Methods for handling this are given in Reference [2].

\section{NUMERICAL EXAMPLES}

We have chosen a few examples of our calculations to illustrate the potential of the method and to check its performance. One objective of these examples is to illustrate how these methods can simplify the modelling of certain engineering problems, such as bearings, joints and bolts, which are very awkward with conventional slideline technology. To model a bolt and nut with conventional methods, the two threads would have to be meshed separately and then interfaced across a slideline. In general, large forces would be generated in turning the bolt due to incompatibilities between the two models. With these new methods, the threads can be modelled by interior discontinuities; this is illustrated for a journal bearing. The methodology for modelling arbitrary discontinuities is called X-FEM (extended finite element method). 


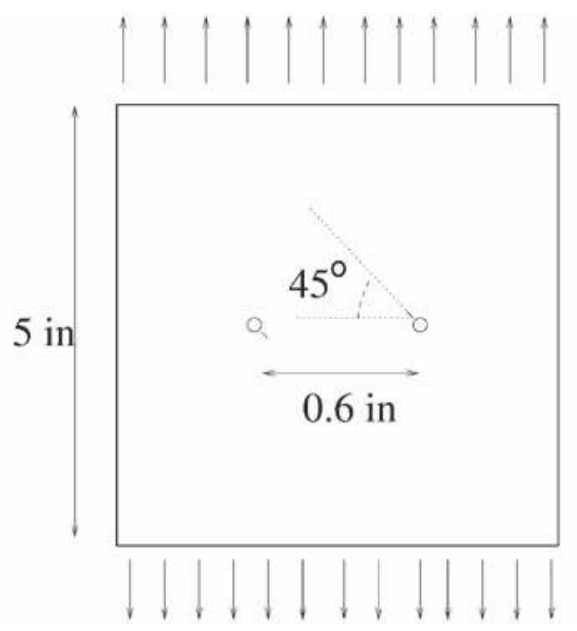

Figure 8 . The problem of two cracks of emanating from rivets holes.

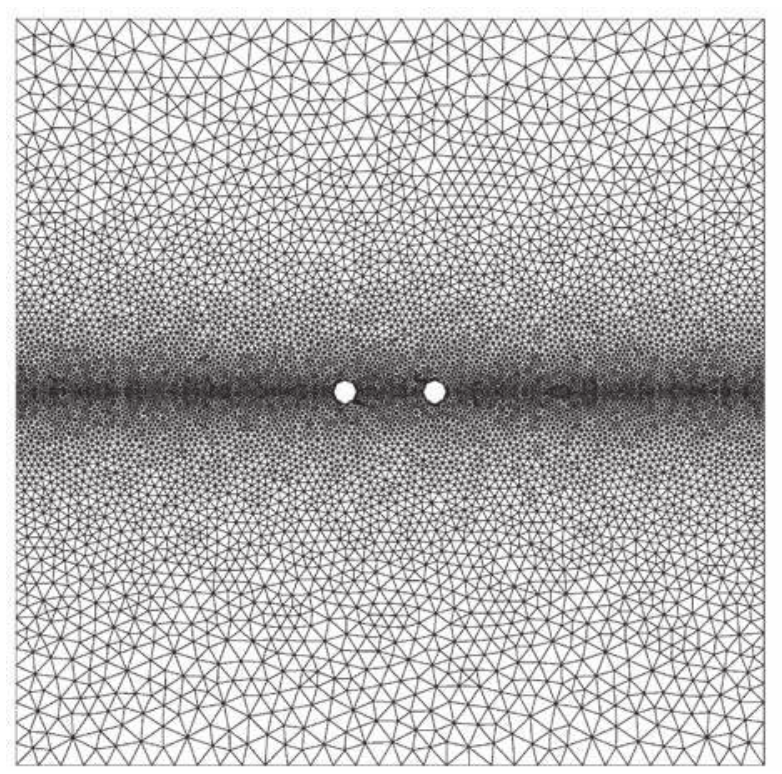

Figure 9. Finite element mesh for the crack propagation problems.

\subsection{Crack propagation problem}

This example concerns crack growth in a plate with two holes. The problem is similar to the one in Reference [2], except that the initial cracks are shorter. The problem is shown in Figure 8 . The ability to model shorter cracks originates from the new definition of the angle $\theta$ in Equation (9). In the initial configuration, the cracks are 0.015 in long and are oriented at $\theta=-45$ and $45^{\circ}$ for the left and right holes, respectively. The plate is a square with the length of each side being 5 in. The rivets of radius 0.08 in are 0.6 in apart. The plate is subject to a cyclic tension in the $y$-direction.

The initial mesh consists of scattered nodes yielding 18,400 triangular elements shown in Figure 9. The mesh is refined to the centre of the plate to obtain more accuracy in the domain of crack growth. The mesh is independent of the crack geometry and the same mesh is used throughout the simulation.

The cracks are driven by the Paris fatigue law with the maximum circumferential stress hypothesis for the direction of propagation. The crack paths are shown in Figure 10. Note that the crack path are completely independent of the finite element mesh.

\subsection{Journal bearing}

The second example is a model of a journal bearing. The model is shown in Figure 11. On the circle marked $\Gamma^{F}$, a discontinuity in the tangential displacement is allowed in the model by adding the enrichment (21). We examine how closely the model can capture the lowest eigenvalue and the eigenvector corresponding to rigid-body rotation of the shaft $\Omega_{\mathrm{A}}$ inside the bearing $\Omega_{\mathrm{B}}$. The lowest eigenvalue should be zero since the shaft $\Omega_{\mathrm{A}}$ should be free to rotate.

When the enrichment (21) is used with Young's modulus $E=1$, the lowest eigenvalue is $5.6 \times 10^{-4}$. Studies of the strain energy distribution associated with the modes show some straining in the vicinity of the interface. Although this result is adequate for many engineering purposes, better results are desirable. 

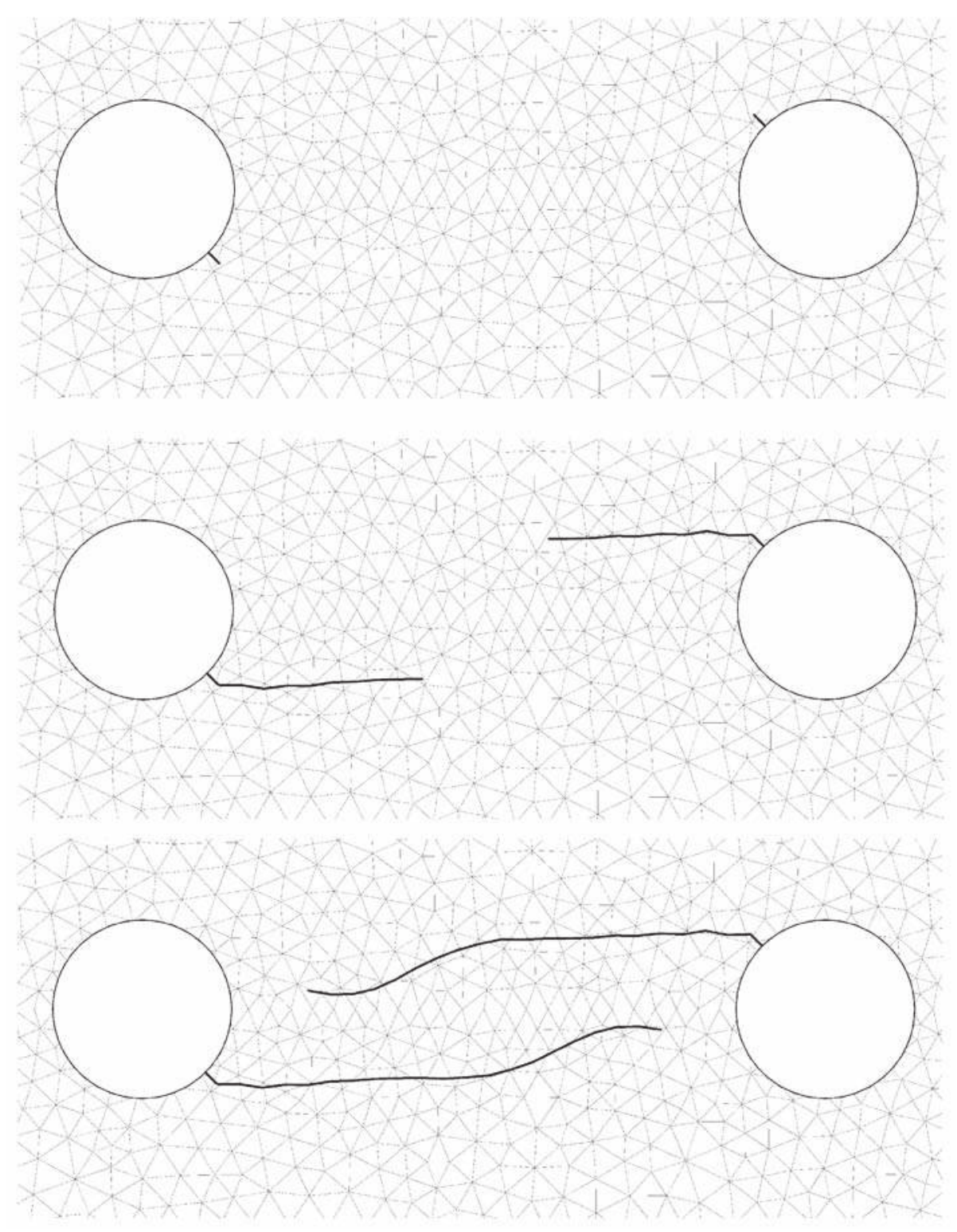

Figure 10. Initial crack and two snapshots of the crack growth.

The accuracy can be improved by adding an enrichment based on the nodal values of the tangent. The enriched approximation is

$$
\mathbf{u}(\mathbf{x})=\sum_{I} N_{I}(\mathbf{x})\left(\mathbf{u}_{I}+a_{I} H(f(\mathbf{x})) \mathbf{v}\left(\mathbf{x}_{I}\right)\right)
$$

where

$$
\mathbf{v}=-\left(y-y_{0}\right) \mathbf{e}_{x}+\left(x-x_{0}\right) \mathbf{e}_{y}
$$

where $x_{0}, y_{0}$ are the co-ordinates of the centre of the shaft of radius $R$ and

$$
f(\mathbf{x})=\left(x-x_{0}\right)^{2}+\left(y-y_{0}\right)^{2}-R^{2}
$$




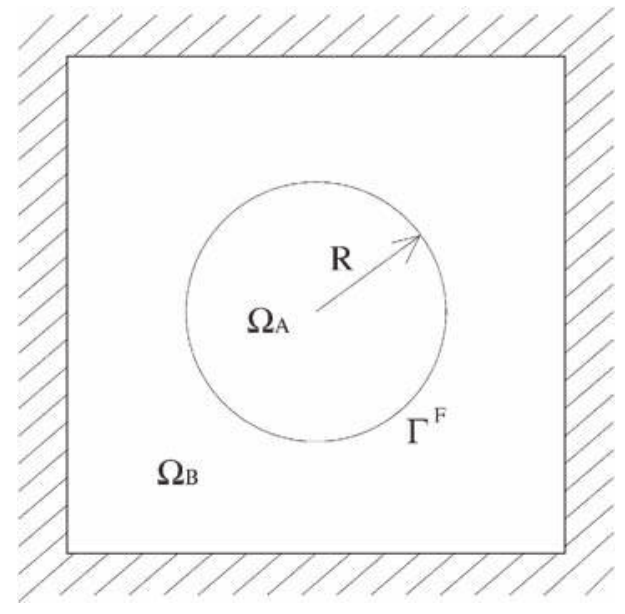

Figure 11. Model of journal bearing.

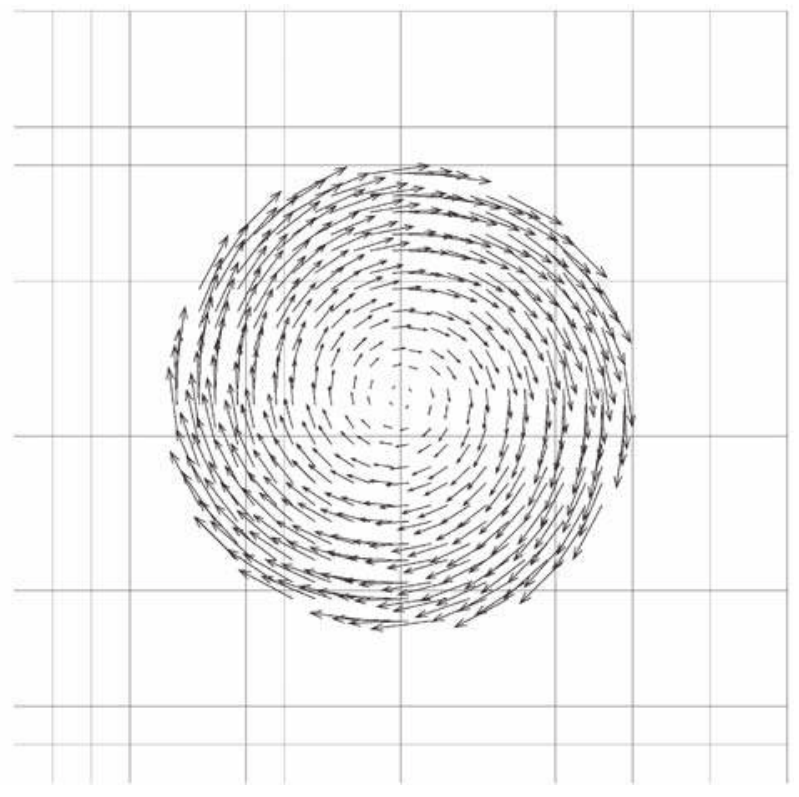

Figure 12. Displacement mode corresponding to the lowest eigenvalue $(\lambda=0.0)$.

The lowest eigenvalue is $4.0 \times 10^{-15}$, which corresponds to zero for the precision used in the calculation. The eigenvector is shown in Figure 12 and can be seen to correspond to rigid-body rotation of the shaft.

\subsection{Circular inclusion under compression}

We consider a circular inclusion in a matrix. The inclusion is separated from the matrix by a frictionless interface without adhesion. The inclusion and the matrix are the same material. The problem has been solved with a combination of Airy stress functions and finite Fourier transforms by Keer et al. (1973). A schematic of the problem is shown in Figure 13. On the interface between the matrix and inclusion, there is no adhesion or friction, so the normal interface traction is either compressive or vanishes; the latter corresponds to separation of the surfaces.

The tractions on the matrix are given by $t_{x}=1, t_{y}=-1$. The material properties are Young's modulus $E=1$ and Poisson's ratio $v=0.0$ for both the matrix and the inclusion. The finite element mesh is shown in Figure 14. The heavy line which separates the inclusion from the matrix is a line of discontinuity either in the total displacement or in the tangential displacement (when the bodies are in contact). The line of discontinuity is independent of the mesh and is a circle (the shape functions are not used to approximate the distance function).

The computed normal stress across the interface is shown in Figure 15 and compared to Keer et al. [17]. The agreement is quite good except near the point of separation. Some of the error is attributed to the fact that the stresses were computed at quadrature points which are not exactly on the interface. 


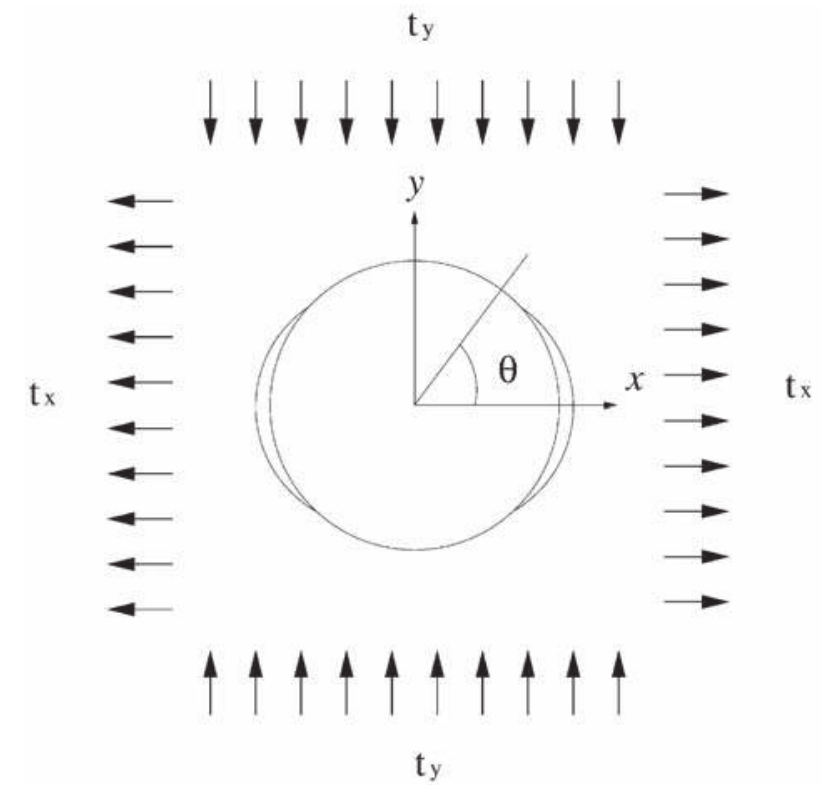

Figure 13. A schematic of the circular inclusion and the load $t_{x}=1$ and $t_{y}=-1$.

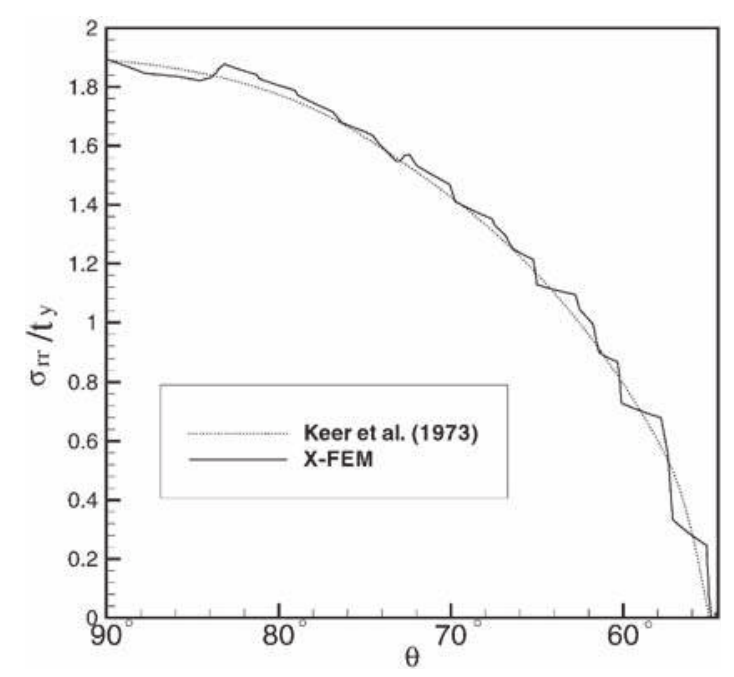

Figure 15. Comparison of normal interface stress for inclusion problem.

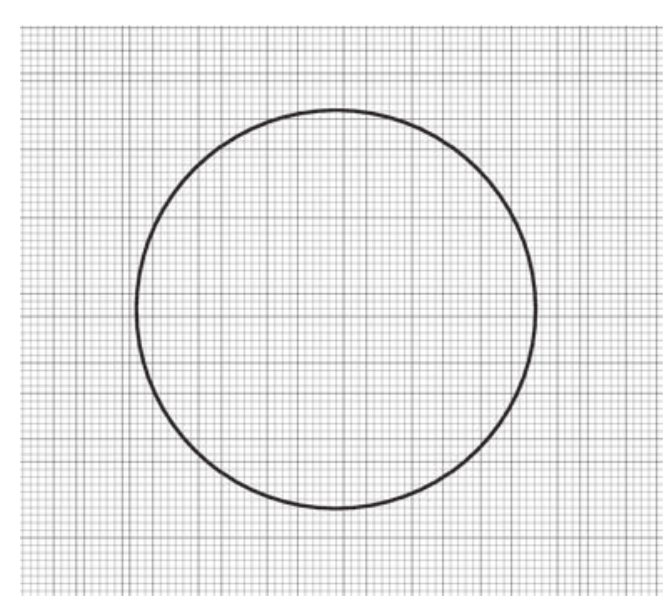

Figure 14. The mesh used for the circular inclusion.

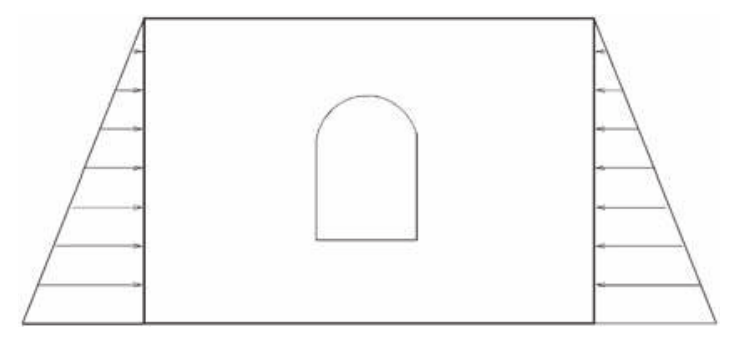

Figure 16. Schematic of the jointed rocks problem from Belytschko et al. [18].

\subsection{Jointed rock mass}

Another example of the application of the methodology is a tunnel in a jointed rock. A schematic of the problem, a scaled model of a tunnel, is shown in Figure 16. This problem was previously studied in Reference [18].

The finite element mesh is shown in Figure 17. Note that the finite element mesh is quite regular and is independent of the orientation of the joints. The mesh extends across the tunnel and the techniques described in Reference [3], in which the quadrature is omitted in any opening, is used. 


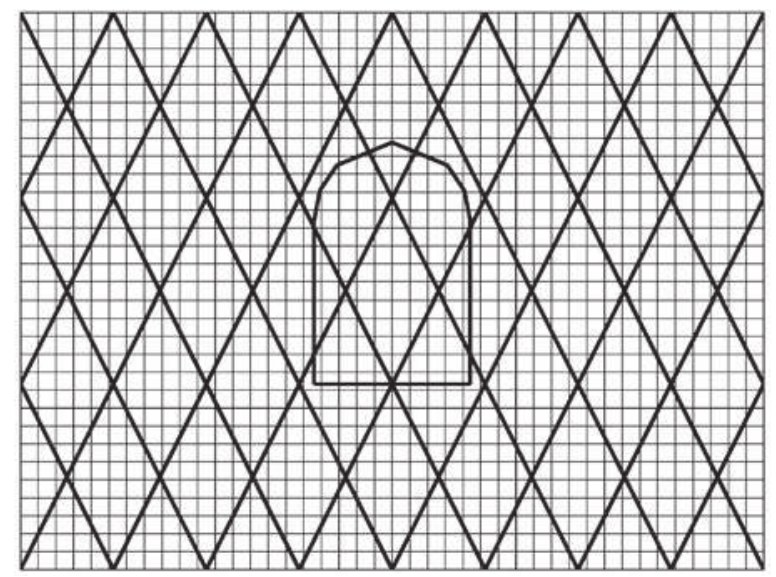

Figure 17. The mesh for the jointed rock problem and the location of joints; the tunnel opening is modelled by excluding it from the quadrature as in Reference [3].
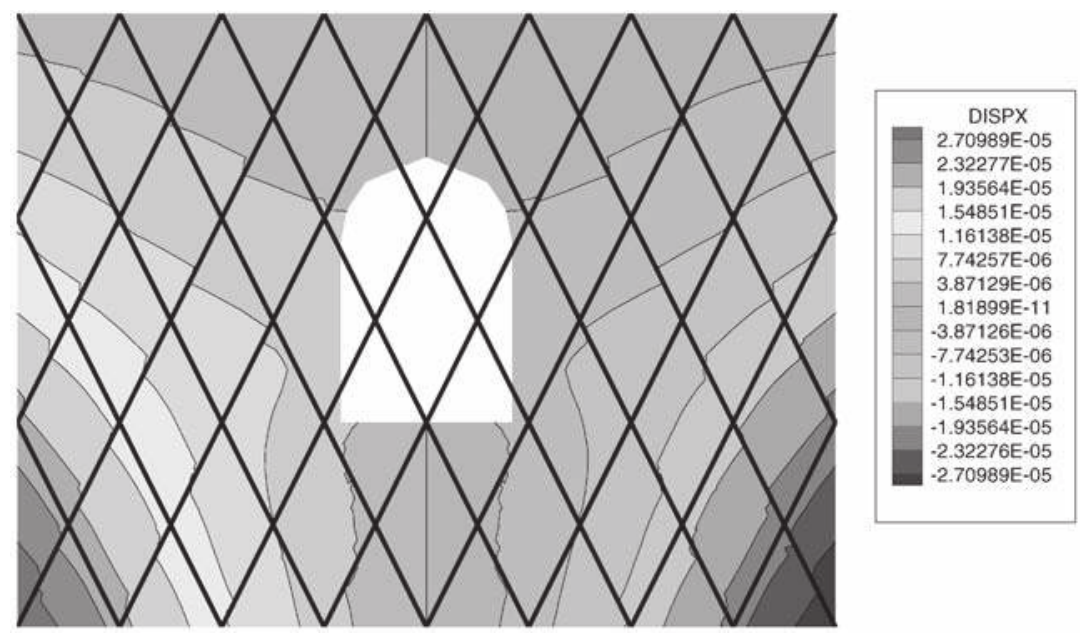

Figure 18. Horizontal component of the displacement field.

A static linear analysis of the problem was performed. In this case, the joints were modelled as tangential discontinuity in the displacement. A tangential stiffness was added at the interfaces as in Reference [18]. In a non-linear analysis it would also be possible to allow separation at the joints. A displacement contour plot of the $x$-component is shown in Figure 18. Note that the displacement is discontinuous across the joints.

\section{DISCUSSION AND CONCLUSIONS}

We have generalized a methodology for representing discontinuities in finite elements which are independent of the mesh. The surface of discontinuity is described by a signed distance function so that the representation of the discontinuous approximation involves only nodal data. Furthermore, this description of the discontinuity leads naturally to a coupling with level set theory for updating the position of the discontinuity. 
The major drawback of the method lies in the quadrature of the weak form. The quadrature of elements in which the discontinuity occurs requires modification to account for the discontinuity. As indicated previously, we have used subdivision of the elements, which is a little bit awkward. Simpler techniques would be desirable.

As examples of applications of these techniques we have shown results for crack growth, a journal bearing, a non-bonded inclusion and a jointed rock mass. There are many others applications: phase changes, shock tracking and fluid-solid interfaces are some examples. Because of its simplicity, the method is very appealing and has considerable potential for problems with stationary and evolving discontinuities without remeshing.

\section{ACKNOWLEDGEMENTS}

The support of the Office of Naval Research to Northwestern University is gratefully acknowledged.

\section{REFERENCES}

1. Belytschko T, Black T. Elastic crack growth in finite elements with minimal remeshing. International Journal for Numerical Methods in Engineering 1999; 45(5):601-620.

2. Moës N, Dolbow J, Belytschko T. A finite element method for crack growth without remeshing. International Journal for Numerical Methods in Engineering 1999; 46:131-150.

3. Daux C, Moës N, Dolbow J, Sukumar N, Belytschko T. Arbitrary branched and intersecting cracks with the extended finite element method. International Journal for Numerical Methods in Engineering 2000; 48: $1741-1760$.

4. Sukumar N, Chopp D, Moës N, Belytschko T. Modeling holes and inclusions by level sets in the extended finite element method. International Journal for Numerical Methods in Engineering 2000; 48:1549-1570.

5. Belytschko T, Lu YY, Gu L. Element-free Galerkin methods. International Journal for Numerical Methods in Engineering 1994; 37:229-256.

6. Oliver J. Continuum modelling of strong discontinuities in solid mechanics using damage models. Computational Mechanics 1995; 17:49-61.

7. Oliver J. Modelling strong discontinuities in solid mechanics via strain softening constitutive equations. Part 2: Numerical simulation. International Journal for Numerical Methods in Engineering 1996; 39:3601-3623.

8. Armero F, Garikipati K. Analysis of strong-discontinuities in inelastic solids with applications to the finite element simulation of strain localization problems. Proceedings of Engineering Mechanics 1996; 1:136-139.

9. Duarte CA, Hamzeh ON, Liszka TJ, Tworzydlo WW. The element partition method for the simulation of threedimensional dynamic crack propagation. Computer Methods in Applied Mechanics and Engineering 2001, submitted for publication.

10. Sethian JA. Level Set Methods and Fast Marching Methods: Evolving Interfaces in Computational Geometry, Fluid Mechanics, Computer Vision, and Materials Sceince. Cambridge University Press: Cambridge, UK, 1999.

11. Rao VS, Hughes TJ, Garikipati K. On modelling thermal oxidation of silicon II: numerical aspects. International Journal for Numerical Methods in Engineering 2000; 47(1):359-377.

12. Stolarski M, Chopp DL, Möes N, Belytschko T. Modelling crack growth by level sets and the extended finite element method. International Journal for Numerical Methods in Engineering 2001, to be published.

13. Melenk JM, Babuška I. The partition of unity finite element method: basic theory and applications. Computer Methods in Applied Mechanics and Engineering 1996; 39:289-314.

14. Krongauz Y, Belytschko T. EFG approximation with discontinuous derivatives. International Journal for Numerical Methods in Engineering 1998; 41(7):1215-1233.

15. Belytschko T, Liu W, Moran B. Nonlinear Finite Elements for Continua and Structures. Wiley: New York, 2000.

16. Strouboulis T, Copps K, Babuška I. The generalized finite element method: an example of its implementation and illustration of its performance. International Journal for Numerical Methods in Engineering 2000; 47(8): 1401-1417.

17. Keer L, Dundurs J, Kitattikomol. Separation of a smooth circular inclusion from matrix. International Journal of Engineering Science 1973; 11:1221-1233.

18. Belytschko T, Plesha M, Dowding C. A computer method for stability analysis of caverns in jointed rock. International Journal for Numerical and Analytical Methods in Geomechanics 1984; 8:473-492. 